\title{
Assessing uncertainty in climate simulations
}

\begin{abstract}
To the Editor - In their Commentary
"Quantifying climate change - too rosy a picture?", Schwartz et al. ${ }^{1}$ suggest that the estimated uncertainties associated with future warming presented in the Intergovernmental Panel on Climate Change Fourth Assessment Report (AR4) ${ }^{2}$ do not correspond to the
\end{abstract} AR4 assessment of uncertainties associated with radiative forcing (that is, a measure of the influence that a factor has in altering the Earth's balance of energy and thereby causing warming or cooling of surface temperatures). In doing so, they criticize estimates of climate sensitivity (that is, the increase in global mean surface temperature expected from a doubling of atmospheric $\mathrm{CO}_{2}$ levels) and estimates of future warming. However, they have misinterpreted the use of AR4 Fig. SPM4 (shown here as Fig. 1) and, as such, their criticisms are misplaced. In the following, we summarize key aspects of the AR4 uncertainty analyses that were neglected by Schwartz et al.

AR4 Fig. SPM-4 illustrates that no model simulation has reproduced observed warming by natural forcing alone for any continent (note that the data for Antarctica were insufficient to make an assessment for the AR4). Atmosphere/Ocean Global Climate Models (AOGCMs), however, are able to reproduce the observed warming on global and continental scales when taking historical natural and anthropogenic forcing into account.

It is clear that the twentieth century climate simulations in AR4 Fig. SPM-4 do not span the full range of uncertainty in radiative forcing or climate sensitivity. As Schwartz et al. point out, and as is well known, models with differing sensitivities and forcings can produce similarly skilful twentieth century simulations. However, the AR4 assessment did not derive estimates of climate sensitivity, of future warming, or of past warming attributable to greenhouse gases directly from these simulations ${ }^{2}$. Instead, uncertainty ranges for climate system properties such as climate sensitivity ${ }^{3,4}$ were derived from many sources including constraints from observed climate change and from studies that systematically varied uncertain model parameters and forcing ${ }^{4}$, the latter often covering or extending beyond the uncertainty range from the AR4 assesment (ref. 5).

Many estimates separate the observed temperature change caused by greenhouse gases from that caused by aerosols using, for example, temporal changes in aerosols ${ }^{4}$ and difference in warming between the Southern

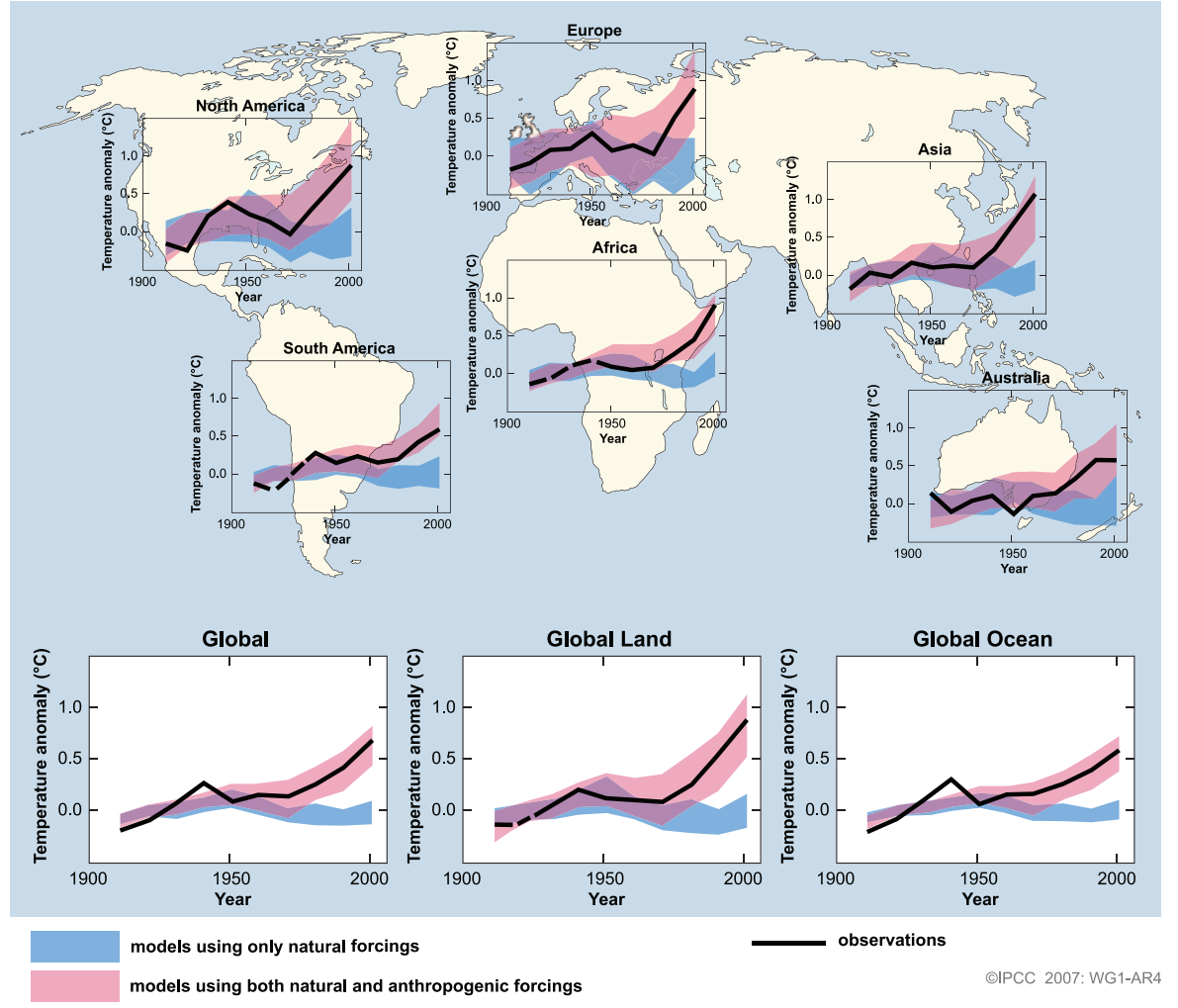

Figure 1. Comparison of observed continental- and global-scale changes in surface temperature with results simulated by climate models using natural and anthropogenic forcings. Decadal averages of observations are shown for the period 1906 to 2005 (black line) plotted against the centre of the decade and relative to the corresponding average for 1901-1950. Lines are dashed where spatial coverage is less than $50 \%$. Blue shaded bands show the $5-95 \%$ range for 19 simulations from five climate models using only the natural forcings due to solar activity and volcanoes. Red shaded bands show the $5-95 \%$ range for 58 simulations from 14 climate models using both natural and anthropogenic forcings.

and Northern Hemisphere, which is partly a response to different amounts of aerosols in each region. In contrast to the suggestion of Schwartz et al. global climate model estimates are just one of many sources of information on climate sensitivity that are accounted for in the AR4 "likely $2-4.5^{\circ} \mathrm{C}$ " range ${ }^{3}$. Indeed, the AR4 assessed the climate sensitivity to have up to a $33 \%$ chance of being outside the range of the global climate models.

In the AR4 assessment, "likely" ranges for future changes in global mean temperature also result from the consideration of multiple sources of uncertainty. This is reflected by the grey ranges in AR4 Fig. SPM- 5 (shown here as Fig. 2), which indicate the likely warming in 2100 depending on which emissions scenario is followed. These ranges are substantially wider than those of individual global climate models ${ }^{2,3}$ depicted by the coloured bands. Nevertheless, despite wide uncertainty ranges in the rate of warming in response to forcing (transient climate response) and in the total amount of forcing, some combinations of transient climate response and forcing are inconsistent with the observed record because they would yield too strong or too weak overall warming, or the wrong time-evolution or spatial pattern of warming. This has been used to narrow the range of uncertainty in future warming presented in the AR4.

A further problem in the analysis of Schwartz et al. is that they assume the uncertainties associated with forcing and with temperature response at year 2000 are linearly related (Schwartz et al. Figure 2). Such a simple relationship does not exist because temperature today is not in equilibrium with current forcing, but instead is influenced by historical forcing. Furthermore, ocean heat uptake is likely to be different for each model. Additionally, Schwartz et al. ignore that the twentieth century model results in their Fig. 2 


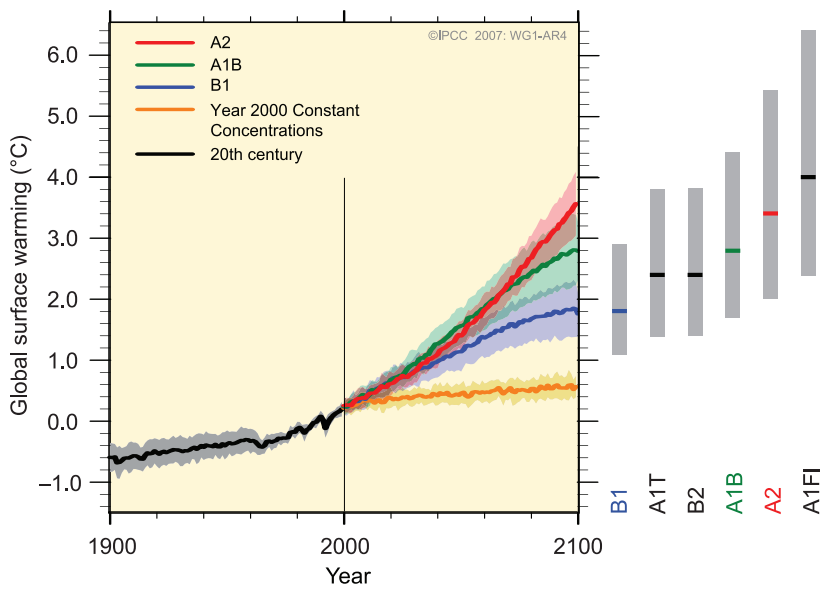

Figure 2. Solid lines are multi-model global averages of surface warming (relative to 1980-1999) for the scenarios A2, A1B and B1, shown as continuations of the 20th century simulations. Shading denotes the \pm 1 standard deviation range of individual model annual averages. The orange line is for the experiment where concentrations were held constant at year $\mathbf{2 0 0 0}$ values. The grey bars at right indicate the best estimate (solid line within each bar) and the likely range assessed for the six SRES marker scenarios. The assessment of the best estimate and likely ranges in the grey bars includes the AOGCMs in the left part of the figure, as well as results from a hierarchy of independent models and observational constraints.

are compared with a 1901-1950 baseline specific to each model. The model range for the year 2000 is therefore smaller than if the models had been compared with the baseline year of 1750 , which is used for estimates of radiative forcing.

We conclude that the skill of climate models in reproducing warming over the twentieth century shown in AR4 does not imply that the IPCC has under-estimated uncertainty in future warming. Uncertainty ranges are not based on the ability of the multi-model ensemble to reproduce twentieth century global mean warming. Instead, the AR4 assessment of future warming provides important new probabilistic information that incorporates multiple sources of uncertainty, including observational data.

Piers Forster ${ }^{1}$, Gabriele Hegerl ${ }^{2 *}$, Reto Knutti ${ }^{3}$, V. Ramaswamy ${ }^{4}$, Susan Solomon ${ }^{5}$, Thomas F. Stocker $^{6}$, Peter Stott ${ }^{7}$ and Francis Zwiers ${ }^{8}$
${ }^{I}$ School of Earth and Environment, University of Leeds, LS2 9JT, UK

${ }^{2} S$ chool of GeoSciences, University of Edinburgh, Grant Institute, W. Mains Road, Edinburgh, Scotland, EH9 3JY, UK

${ }^{3}$ Institute for Atmospheric and Climate Science, Universitätstrasse 16, ETH Zürich, CHN, CH-8092 Zürich, Switzerland

${ }^{4}$ Geophysical Fluid Dynamics Laboratory, Princeton University Forrestal Campus, 201 Forrestal Road, Princeton, New Jersey 08540-6649, USA ${ }^{5}$ NOAA Earth System Research Laboratory, 325 Broadway, Boulder, Colorado 80305, USA

${ }^{6}$ Climate and Environmental Physics, Physics Institute, University of Bern, Sidlerstrasse 5, 3012 Bern, Switzerland

${ }^{7}$ Met Office Hadley Centre (Reading Unit), Meteorology Building, University of Reading, Reading RG6 6BB, UK

${ }^{8}$ Climate Research Division, Environment Canada, Toronto, Ontario M3H 5T4, Canada.

"e-mail: gabi.hegerl@ed.ac.uk

References

1. Schwartz, S.E., Charlson, R.J., \& Rhode, H. Nat. Rep. Clim. Change 1, 23-24 (2007)

2. Solomon, S. et al. (eds) Climate Change 2007: The Physical Science Basis (Cambridge Univ. Press for the Intergovernmental Panel on Climate Change, Cambridge, 2007).

3. Meehl, G. A. et al. in Climate Change 2007: The Physical Science Basis (eds Solomon, S. et al.) Ch. 10 (Cambridge Univ. Press for the Intergovernmental Panel on Climate Change, Cambridge, 2007).

4. Hegerl, G. C. et al. in Climate Change 2007: The Physical Science Basis (eds Solomon, S. et al.) Ch. 9 (Cambridge Univ. Press for the Intergovernmental Panel on Climate Change, Cambridge, 2007).

5. Forster, P. et al. in Climate Change 2007: The Physical Science Basis (eds Solomon, S. et al.) Ch. 2 (Cambridge Univ. Press for the Intergovernmental Panel on Climate Change, Cambridge, 2007).
Authors' response - In their reply to our Commentary ${ }^{1}$, Forster et al. ${ }^{2}$ mainly criticize us for points which we did not make regarding climate sensitivity and future global warming and fail to come to grips with our central point, namely that in assessing the skill of climate models by their ability to reproduce warming over the twentieth century, the latest report from the IPCC ${ }^{3}$ may give a false sense of their predictive capability. Increasing the spread of the modelled twentieth century climate change may have implications for estimates of climate sensitivity and its uncertainty and for projections of future climate change, but those implications cannot be determined unless the consequences of uncertainties in forcing are systematically examined.

Additionally, we have several specific points in response to Forster et al. as follows: - Changes in forcing over time that are scaled by a constant factor would, for a linear relationship between forcing and response, result in a relative change in surface temperature of the same factor irrespective of the time period over which the temperature change was measured. There would thus seem to be little justification for relying on the modelled temperature change not responding linearly to the applied forcing or the temperature change not being in equilibrium with the forcing as the reason for reduction in uncertainty in temperature change relative to that of forcing in the absence of model experiments explicitly examining these points. - No comfort should be taken in the fact that different models used in the study may exhibit different rates of ocean heat uptake; rather the goal of stringent examination of model performance should be to rule out, or improve, those models that poorly represent the process being simulated.

- We did not dispute that forced simulations are necessary for models to exhibit the observed increases in surface temperature.

Climate models are essential for examining prospective future climate change that would result from changes in atmospheric composition and other forcings. We anticipate that much progress would be made in understanding and characterizing the present suite of climate models if the IPCC and modelling groups that participated in the exercise leading to Fig. SPM-4 (ref. 3) were to systematically examine the implications of the uncertainty in forcing. Only by constraining the forcing will it be possible to rule out models that skilfully reproduce twentieth century warming for the wrong reason.

\section{S. E. Schwartz ${ }^{1 \star}$, R. J. Charlson ${ }^{2}$ and H. Rodhe ${ }^{3}$}

${ }^{1}$ Atmospheric Sciences Division, Brookhaven National Laboratory, Upton, New York 11973, USA ${ }^{2}$ Department of Atmospheric Sciences, University of Washington, Seattle, Washington 98195, USA ${ }^{3}$ Department of Meteorology, Stockholm University, 10691 Stockholm Sweden

*e-mail: ses@bnl.gov

References

1. Schwartz, S. E., Charlson R. J. \& Rodhe H. Nat. Rep. Clim. Change 1, 23-24 (2007).

2. Forster P. et al. Nat. Rep. Clim. Change 1, 63-64 (2007).

3. Solomon, S. et al. (eds.) Climate Change 2007: The Physical Science Basis. (Cambridge Univ. Press for the Intergovernmental Panel on Climate Change, Cambridge, UK, 2007). 